\title{
Estrutura e composição do estrato de regeneração em um fragmento florestal urbano: implicações para a dinâmica e a conservação da comunidade arbórea
}

\author{
Jania Cabrelli Salles ${ }^{1}$ e Ivan Schiavini ${ }^{2}$
}

Recebido em 8/05/2005. Aceito em 11/09/2006

\begin{abstract}
RESUMO - (Estrutura e composição do estrato de regeneração em um fragmento florestal urbano: implicações para a dinâmica e a conservação da comunidade arbórea). Este estudo foi realizado em um fragmento de floresta estacional semidecídua, sujeito a longo histórico de perturbação antrópica, localizado no perímetro urbano de Uberlândia, MG. Teve como objetivo avaliar a composição e a estrutura do estrato regenerativo e comparar com a comunidade adulta do dossel, de forma a revelar informações importantes sobre o funcionamento do ambiente e a dinâmica dessa formação vegetal. Para o levantamento do estrato regenerativo, utilizaram-se 30 parcelas de $10 \times 10 \mathrm{~m}$, onde todos os indivíduos com altura igual ou superior a um metro tiveram medidos a altura e o diâmetro ao nível do solo. A presença de espécies tardias de grande porte no estrato adulto, sem a correspondência de abundância no estrato regenerativo, sugere que as perturbações atingem diretamente o estrato inferior. O dossel da floresta estacional do Parque do Sabiá é composto de árvores que, provavelmente, possuem dezenas de anos e a formação de clareiras, naturais ou antrópicas, estimula o estabelecimento de espécies pioneiras. De forma geral, as espécies importantes do estrato regenerativo sugerem que a comunidade está sofrendo mudanças na sua composição florística, em um processo de sucessão caracterizado pela presença de grande número de indivíduos regenerantes, de espécies pertencentes aos primeiros estágios sucessionais.
\end{abstract}

Palavras-chave: regeneração natural, floresta estacional semidecídua, perturbação antrópica, fitossociologia

\begin{abstract}
Structure and composition of the regeneration layer in an urban forest fragment: implications for the dynamics and conservation of the tree community). This study was carried out in a semideciduous seasonal forest fragment with a long history of manmade disturbance, located in urban Uberlândia, Minas Gerais. The aim was to compare the composition and structure of the regeneration layer with that of the adult canopy layer in order to obtain information on environmental functioning and the dynamics of this plant formation. In 30 plots of $10 \times 10 \mathrm{~m}$, plants equal to or taller than one meter were surveyed, recording height and stem diameter at soil level. The presence of large trees of secondary species in the canopy layer, without corresponding abundance in the regeneration layer, suggests that disturbance affects the understory directly. The canopy of the seasonal forest at Parque do Sabiá is composed of trees probably decades old; natural or manmade gap formation stimulates the establishment of pioneer species. In general, the important species of the regeneration layer suggest that the floristic composition of the community is changing in a process of succession characterized by the presence of many young individuals belonging to species of the early successional stages.
\end{abstract}

Key words: natural regeneration, semideciduous seasonal forest, manmade disturbance, phytosociology

\section{Introdução}

O aumento descontrolado na taxa de conversão das Florestas Tropicais em fragmentos florestais tem como resultados a perda de espécies, a mudança da composição florística ao longo do tempo (Nascimento et al. 1999; Cielo Filho \& Santin 2002), a alteração das interações entre animais e plantas, como a polinização e a dispersão de sementes (Aizen \& Feinsinger 1994; Santos \& Tellería 1994; Galetti et al. 2003), entre outras conseqüências. A intensidade na qual ocorre a deterioração de uma paisagem composta por fragmentos depende das características do ambiente no qual estão inseridos; logo o tamanho, a forma e o tipo de vizinhança influenciam no número de espécies capazes de sobreviver em um fragmento (Saunders et al. 1991; Viana et al. 1992; Nascimento et al. 1999).

O desenvolvimento de estudos com enfoque nas características biológicas e ecológicas de paisagens fragmentadas visa traçar um perfil do seu funcionamento e estabelecer medidas racionais de manejo e preservação (Pagano \& Leitão Filho 1987; Pereira et al. 2001). Os estudos de comunidades vegetais afetadas pelo desenvolvimento das atividades humanas relacionadas à urbanização são importantes

\footnotetext{
1 Universidade Federal de Uberlândia, Instituto de Biologia, C. Postal 593, 38400-902 Uberlândia, MG, Brasil

2 Autor para correspondência: schiavini@ufu.br
} 
instrumentos para a manutenção desses frágeis ecossistemas. É imprescindível conhecer, por exemplo, como se dão os processos relacionados à dinâmica, à sucessão ecológica e à regeneração natural diante das perturbações antrópicas (Pereira et al. 2001).

O estudo de fenômenos relacionados às variações na estrutura das comunidades tem, cada vez mais, envolvido indivíduos que já tenham ultrapassado o período crítico de mortalidade, por representarem o potencial regenerativo da estrutura arbórea (MartinezRamos 1991; Felfili et al. 2000; Oliveira et al. 2001). De acordo com Felfili et al. (2000), estrato regenerativo refere-se aos indivíduos com altura igual ou superior a um metro, que representam o potencial regenerativo da comunidade arbórea, por já terem superado a forte ação seletiva do ambiente e, assim, já ultrapassaram o período crítico de mortalidade.

A avaliação do potencial regenerativo de um ecossistema deve descrever os padrões da substituição das espécies ou das alterações estruturais, bem como os processos envolvidos na manutenção da comunidade (Guariguata \& Ostertag 2001). Conhecer a composição e a estrutura florística do estrato regenerativo, que já tenha superado a forte ação seletiva do ambiente, e a posterior comparação desse estrato com a estrutura da comunidade adulta pode trazer respostas instantâneas sobre a dinâmica ambiental (Mendes 2002).

Com base nisso, este estudo teve como objetivo avaliar a composição e a estrutura do estrato regenerativo de um fragmento florestal urbano e comparar com a comunidade adulta do dossel, o que revelou informações importantes sobre o funcionamento do ambiente e a dinâmica dessa formação vegetal. Além disso, foi possível avaliar o estado de conservação desse fragmento florestal submetido a diferentes tipos e graus de perturbação.

\section{Material e métodos}

O Parque Municipal do Sabiá localiza-se no perímetro urbano do município de Uberlândia, Minas Gerais, a cerca de $6 \mathrm{~km}$ do centro da cidade, nas coordenadas $18^{\circ} 54$ '52"S e $48^{\circ} 14^{\prime} 02^{\prime \prime} \mathrm{W}$. O clima da região é do tipo Aw Megatérmico, segundo a classificação de Köppen, caracterizado por apresentar verões chuvosos, com temperatura média mensal entre 21 e $26{ }^{\circ} \mathrm{C}$, e invernos secos, quando a temperatura média mensal varia entre 17 e $22{ }^{\circ} \mathrm{C}$ (Rosa et al. 1991). Na estação chuvosa, a precipitação média mensal pode atingir $333,8 \mathrm{~mm}$ (dezembro), enquanto na estação seca cai para até $12,1 \mathrm{~mm}$ (junho), de acordo com o $5^{\circ}$ Distrito de Meteorologia. Os solos da região onde está inserido o Parque do Sabiá são caracterizados como Latossolo Vermelho - Amarelo Álico (Rosa \& Schiavini 2006). Segundo Baccaro (1989), o relevo é classificado como medianamente dissecado, com topos aplainados entre 700 e $900 \mathrm{~m}$ e vertentes suaves, interrompidas por rupturas locais mantidas pela laterita.

Na região de Uberlândia, as áreas primárias de domínio do Cerrado e suas fisionomias encontram-se restritas a pequenos remanescentes (Araújo \& Haridasan 1997). Dentre as formações que compõem o domínio do Cerrado (sensu Ribeiro \& Walter 1998), a floresta estacional semidecídua (equivalente à mata seca, descrita por Ribeiro \& Walter 1998) é o tipo de formação vegetal que mais rápida e extensamente tem sido destruída na região, sendo representada, atualmente, por fragmentos de diferentes tamanhos e submetidos aos mais variados tipos de perturbação (Araújo \& Haridasan 1997).

Particularmente para o município de Uberlândia, o Parque do Sabiá possui um dos remanescentes de mata nativa de maior importância do âmbito regional, enquadrada como Unidade de Conservação na categoria Parque Municipal, desde 1997. O remanescente possui cerca de 30 ha, caracterizado por apresentar diferentes formações naturais heterogêneas: floresta estacional semidecídua (objeto deste estudo), transição com cerradão e mata de galeria (Guilherme et al. 1998).

Até o ano de 1994, a administração do Parque realizava a limpeza periódica do solo da mata, retirando folhas, sementes e plântulas provenientes do estrato arbóreo e, também, de herbáceas e arbustos, com a finalidade de garantir a segurança dos visitantes e permitir o uso dessa área para lazer e recreação. Além disso, existe grande quantidade de trilhas passando pelo interior da mata e observa-se um aumento gradativo na densidade populacional de uma espécie de bambu invasor (Olyra sp.) no estrato inferior.

O levantamento do estrato arbóreo adulto foi realizado por Rosa \& Schiavini (2006), o qual compreendeu indivíduos com circunferência à altura do peito (CAP) igual ou maior que $15 \mathrm{~cm}$. Nesse levantamento, foram utilizadas 104 parcelas de $10 \times 10 \mathrm{~m}$, instaladas sobre oito transectos, posicionados perpendicularmente ao córrego Jataí, dispostos paralelamente e distantes 50 metros entre si (Fig. 1). Entretanto, os dados originais do estrato arbóreo foram reestruturados, de forma que, das 104 parcelas previamente amostradas por Rosa \& Schiavini (2006), 


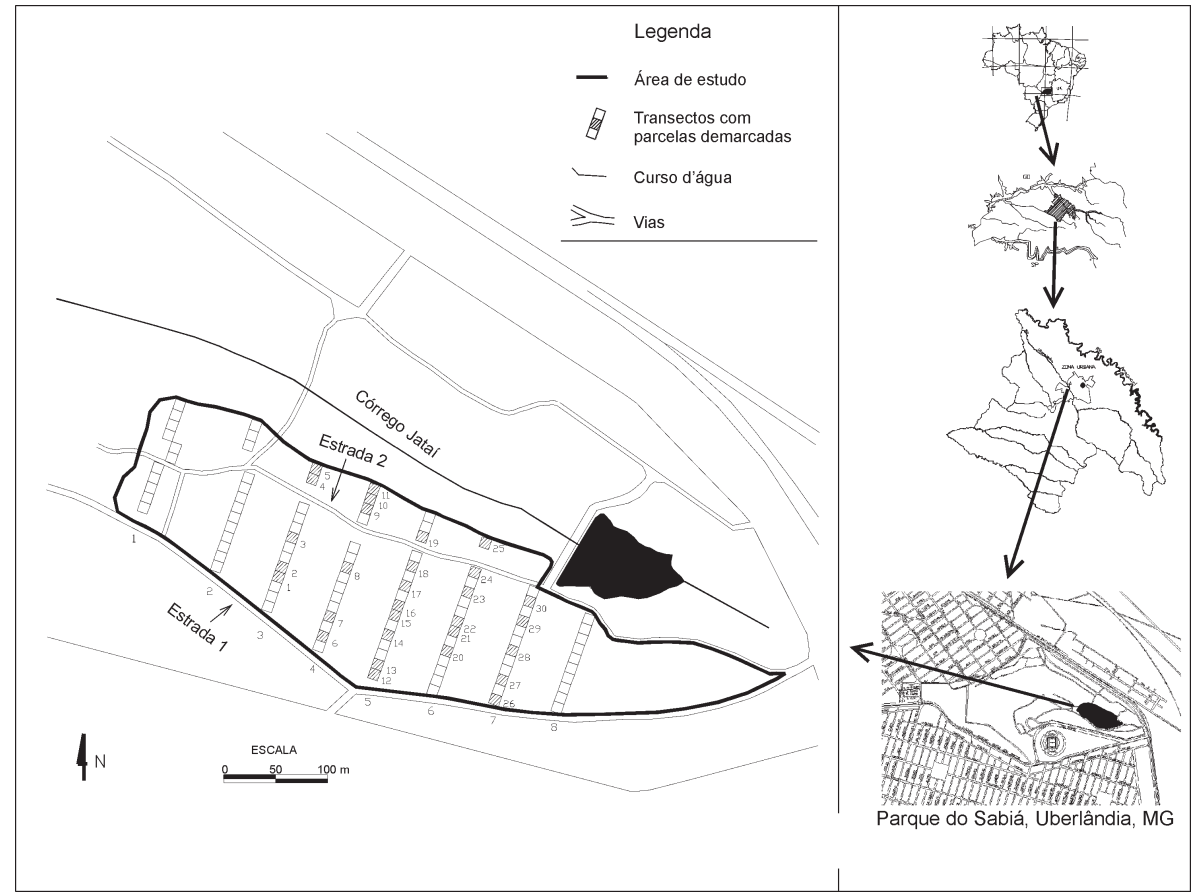

Figura 1. Localização da área de estudo na floresta estacional semidecídua do Parque do Sabiá, Uberlândia, MG.

as 34 parcelas localizadas nos transectos 1,2 e 8 foram excluídas, restando apenas 70 parcelas para análise desenvolvida no presente estudo, ou seja, para a comparação entre o estrato arbóreo adulto e o estrato de regeneração. Além disso, foram excluídos os indivíduos mortos. Após tais modificações, os dados referentes ao estrato arbóreo foram submetidos aos cálculos dos parâmetros fitossociológicos (FITOPAC Shepherd 1995).

Os transectos 1 e 2 foram excluídos, pois os mesmos encontram-se plotados na transição da floresta estacional com o cerradão, cujas parcelas apresentaram Índice de Similaridade de Sorensen menor que $50 \%$ em relação às outras (Rosa \& Schiavini 2006). O transecto 8 também foi desconsiderado por estar localizado próximo à borda da área de estudo.

Para o levantamento fitossociológico do estrato regenerativo, que ocorreu entre outubro/2002 e maio/2003, foram utilizadas parcelas previamente demarcadas para o estudo do estrato arbóreo. Foram sorteadas 30 parcelas de $10 \times 10 \mathrm{~m}$ dentre aquelas localizadas nos transectos 3 a 7 (Fig. 1).

Em cada parcela, todos os indivíduos de espécies arbóreas com altura igual ou superior a um metro, que não foram incluídos no estudo do estrato arbóreo adulto, tiveram medidos altura e diâmetro ao nível do solo. As medidas de altura foram obtidas com auxílio de uma vara graduada de $2 \mathrm{~m}$ ou por estimativa visual, para indivíduos com altura superior a $2 \mathrm{~m}$. O diâmetro foi medido na base do tronco, com o uso de paquímetro. Aqueles indivíduos que apresentavam ramificações ao nível do solo tiveram todos os caules medidos, mesmo aqueles que não alcançavam um metro de altura.

A identificação das espécies foi realizada no campo e, quando isso não foi possível, foram coletadas e posteriormente identificadas por especialistas ou por comparação com exsicatas pertencentes ao acervo do Herbarium Uberlandense (HUFU), da Universidade Federal de Uberlândia. A classificação das espécies seguiu o sistema de Cronquist (1988).

A vegetação do estrato regenerativo foi analisada utilizando-se o programa FITOPAC (Shepherd 1995), a partir do qual foram calculados os parâmetros fitossociológicos (densidade, freqüência e dominância relativas), que permitiram a ordenação das espécies de acordo com o Índice de Valor de Importância (IVI). O Índice de Diversidade de Shannon (H') para espécies também foi calculado, de acordo com Brower et al. (1997).

Também foi calculada a similaridade florística entre os dois estratos, pelo índice de Soørensen (Brower et al. 1997) e a proporção de indivíduos regenerantes em relação aos adultos das 10 espécies mais importantes em cada estrato. O cálculo da proporção teve como referência a densidade absoluta encontrada 
nos dois inventários, estimada para um hectare. Esse procedimento teve como objetivo avaliar as tendências de declínio ou aumento populacional, a fim de verificar a manutenção das espécies na área.

\section{Resultados e discussão}

A compilação de estudos de estrato regenerativo revelou a grande escassez de informações no que se refere à dinâmica e ao potencial regenerativo de áreas de floresta estacional semidecídua pertencentes ao domínio do Cerrado. Dessa forma, para a comparação dos dados obtidos no presente estudo foram utilizados estudos feitos em outras fisionomias, mas, é preciso reconhecer que a heterogeneidade intrínseca de cada formação vegetal, como a dinâmica natural, as características topográficas e as condições de microclima exercem grande influência na determinação dos resultados encontrados. Além disso, ao comparar os valores de densidade e diversidade encontrados em outros estudos, mesmo que em diferentes fisionomias de domínio do Cerrado, deve-se levar em consideração o efeito da ação antrópica sobre a comunidade vegetal do presente estudo.

No levantamento do estrato regenerativo da floresta estacional semidecídua do Parque do Sabiá, foram amostrados 1.268 indivíduos, pertencentes a 63 espécies de 32 famílias, com densidade total estimada de 4.226 ind. ha ${ }^{-1}$. O Índice de Diversidade de Shannon $\left(H^{\prime}\right)$ para espécies foi de 3,10, sendo que 50,62\% dos indivíduos pertencem a apenas cinco espécies (Tab. 1).

$\mathrm{O}$ valor de densidade total estimado para a área de estudo foi considerado baixo, se comparado a outros levantamentos de estrato regenerativo. S. Mendes (dados não publicados), estudando a regeneração de uma mata de galeria na região de Uberlândia, Minas Gerais, encontrou densidade igual a 7.267 ind. ha ${ }^{-1}$. Em outra área de mata de galeria no município de Uberlândia, o estudo do estrato regenerativo, realizado por E. Amorim (dados não publicados), estimou densidade em 12.190 ind. ha ${ }^{-1}$. Durigan et al. (2002), em área de cerrado stricto sensu, em Brotas, São Paulo, encontraram densidade de 10.220 ind. ha ${ }^{-1}$. Também estudando o estrato regenerativo em área de cerrado stricto sensu, Barreira et al. (2002) estimaram a densidade em 14.622 ind. ha ${ }^{-1}$. Entretanto, estes dois últimos trabalhos amostraram indivíduos muito jovens, com menos de um metro de altura. Nessa fase, as plântulas sofrem intensa pressão seletiva do ambiente e, de acordo com Felfili (1997), Felfili \& Abreu (1999) e Felfili et al. (2000), são consideradas ainda "não estabelecidas" e muitas dificilmente atingirão a idade adulta.

O valor da diversidade encontrado para o estrato regenerativo do presente estudo e a comparação com os encontrados no estrato de regeneração de diferentes fisionomias do Cerrado revelam que a distribuição dos indivíduos entre as espécies é variável nas diferentes comunidades. Alguns estudos revelaram H' maior, como Durigan et al. (2002), que encontraram valor de $\mathrm{H}^{\prime}$ igual a 3,32, estudando o estrato inferior em área de cerrado stricto sensu. Outros estudos revelam valores de diversidade abaixo do encontrado. A.F. Rozza (dados não publicados), estudando o estrato regenerativo de mata estacional semidecidual, em Matão, obteve $\mathrm{H}^{\prime}=1,59$, Durigan et al. (2000), também em mata estacional, encontraram $\mathrm{H}^{\prime}=2,01$, e Barreira et al. (2002), em área de cerrado stricto sensu, encontraram índice de 2,90. Segundo Tabarelli \& Mantovani (1999), Werneck et al. (2000) e Rosa \& Schiavini (2006), o método de amostragem, a área total amostrada, os critérios de inclusão escolhidos, o estágio sucessional e o grau de perturbação da área são fatores que influenciam na diversidade, pois levam em consideração a riqueza e a abundância das espécies.

Nas 70 parcelas do estrato arbóreo foram encontrados 636 indivíduos, pertencentes a 71 espécies de 39 famílias, sendo a densidade total estimada em 908 ind.ha ${ }^{-1}$ e o Índice de Diversidade de Shannon (H') calculado, para espécies, de 3,48 (Tab. 2).

Em relação ao Índice de Diversidade (H'), observou-se que o estrato regenerante apresentou valor inferior ao estrato arbóreo. $\mathrm{O}$ menor $\mathrm{H}^{\prime}$ do estrato regenerativo do Parque do Sabiá pode ser decorrente da alta densidade apresentada por pequeno número de espécies, além de revelar um efeito das perturbações, refletindo nos valores de riqueza e abundância.

Em florestas tropicais, a riqueza florística é um dos primeiros atributos a serem restaurados no processo sucessional, sendo que a velocidade de recuperação depende da presença de plântulas, banco e chuva de sementes (Brown \& Lugo 1990; Tabarelli \& Mantovani 1999; Pascarella et al. 2000; Aide et al. 2000; Guariguata \& Ostertag 2001). No caso da floresta do Parque do Sabiá, a prática de limpeza do sub-bosque (até 1994) e o alto grau de pisoteio (ainda ocorrente, sobretudo, nas inúmeras trilhas), podem influenciar na velocidade, ou mesmo na possibilidade, de recuperação do número de espécies e na participação dos diferentes grupos ecológicos na estrutura de comunidade. 
Tabela 1. Estrutura fitossociológica do estrato regenerativo da floresta estacional semidecídua do Parque do Sabiá, Uberlândia, MG, em ordem decrescente do Índice de Valor de Importância. $(\mathrm{N}=$ número de indivíduos amostrados; D.R.= densidade relativa; Do. $\mathrm{R} .=$ dominância relativa; F.R.= freqüência relativa; IVI= Índice de Valor de Importância).

\begin{tabular}{|c|c|c|c|c|c|}
\hline Espécie/Família & $\mathrm{N}$ & D. R. & Do. R. & F. R. & IVI \\
\hline Piptocarpha macropoda (DC.) Baker. (Asteraceae) & 187 & 14,75 & 20,07 & 3,96 & 38,77 \\
\hline Ocotea corymbosa (Miers.) Mez (Lauraceae) & 189 & 14,91 & 6,12 & 6,60 & 27,62 \\
\hline Casearia grandiflora Camb. (Flacourtiaceae) & 98 & 7,73 & 10,63 & 5,54 & 23,90 \\
\hline Xylopia aromatica (Lam.) Mart. (Annonaceae) & 65 & 5,13 & 9,10 & 4,47 & 18,71 \\
\hline Ocotea spixiana (Ness) Mez (Lauraceae) & 103 & 8,12 & 3,62 & 5,80 & 17,55 \\
\hline Siparuna guianensis Aubl. (Monimiaceae) & 62 & 4,89 & 6,16 & 3,96 & 15,01 \\
\hline Annona cacans Warm. (Annonaceae) & 71 & 5,60 & 2,55 & 5,80 & 13,96 \\
\hline Maprounea guianensis (Aubl.) M. Arg. (Euphorbiaceae) & 57 & 4,50 & 2,75 & 4,22 & 11,47 \\
\hline Inga vera Willd. (Mimosaceae) & 34 & 2,68 & 4,11 & 3,96 & 10,75 \\
\hline Virola sebifera Aubl. (Myristicaceae) & 34 & 2,68 & 2,74 & 3,69 & 9,11 \\
\hline Micropholis venulosa (Mart. \& Eichl.) Pierre (Sapotaceae) & 39 & 3,08 & 2,60 & 3,43 & 9,11 \\
\hline Miconia cuspidata Naud. (Melastomataceae) & 42 & 3,31 & 2,77 & 2,11 & 8,19 \\
\hline Amaioua guianensis Aubl. (Rubiaceae) & 30 & 2,37 & 1,18 & 3,17 & 6,71 \\
\hline Qualea jundiahy Warm. (Vochysiaceae) & 20 & 1,58 & 1,37 & 3,17 & 6,12 \\
\hline Tapirira obtusa (Benth.) J.D. Mitch. (Anacardiaceae) & 7 & 0,55 & 2,48 & 1,85 & 4,88 \\
\hline Miconia sellowiana Naud. (Melastomataceae) & 16 & 1,26 & 1,39 & 2,11 & 4,76 \\
\hline Astronium nelson-rosae D.A. Santin (Anacardiaceae) & 15 & 1,18 & 1,47 & 1,85 & 4,50 \\
\hline Dalbergia nigra (Vell.Conc.)Benth. (Fabaceae) & 14 & 1,10 & 1,00 & 2,37 & 4,48 \\
\hline Senna macranthera (Collad.) I. \& B. (Caesalpiniaceae) & 9 & 0,71 & 1,15 & 2,11 & 3,98 \\
\hline Cryptocaria aschersoniana Mez (Lauraceae) & 6 & 0,47 & 1,64 & 1,58 & 3,69 \\
\hline Duguetia lanceolata St. Hil. (Annonaceae) & 10 & 0,79 & 1,51 & 1,32 & 3,62 \\
\hline Hirtella glandulosa Spreng. (Chrysobalanaceae) & 8 & 0,63 & 1,34 & 1,58 & 3,55 \\
\hline Hirtella gracilipes (Hook. F.) (Chrysobalanaceae) & 11 & 0,87 & 0,79 & 1,58 & 3,24 \\
\hline Aspidosperma discolor A. DC. (Apocynaceae) & 8 & 0,63 & 0,71 & 1,85 & 3,19 \\
\hline Cardiopetalum calophyllum Schlecht. (Annonaceae) & 8 & 0,63 & 0,47 & 1,85 & 2,95 \\
\hline Myrsine coriacea (Mart.) Mez (Myrsinaceae) & 10 & 0,79 & 0,47 & 1,32 & 2,58 \\
\hline Apuleia leiocarpa (Vog.) Macbr. (Fabaceae) & 7 & 0,55 & 0,68 & 1,32 & 2,56 \\
\hline Coussarea hydrangeaefolia Benth. \& Hook. F. (Rubiaceae) & 11 & 0,87 & 0,58 & 1,06 & 2,50 \\
\hline Callisthene major Mart. (Vochysiaceae) & 13 & 1,03 & 0,42 & 1,06 & 2,50 \\
\hline Vochysia tucanorum Mart. (Vochysiaceae) & 6 & 0,47 & 1,09 & 0,79 & 2,36 \\
\hline Siphoneugenia densiflora Berg. (Myrtaceae) & 3 & 0,24 & 1,18 & 0,79 & 2,20 \\
\hline Matayba guianensis Aubl. (Sapindaceae) & 9 & 0,71 & 0,41 & 1,06 & 2,17 \\
\hline Cupania vernalis Camb. (Sapindaceae) & 5 & 0,39 & 0,32 & 1,06 & 1,77 \\
\hline Pseudolmedia laevigata Tréc. (Moraceae) & 8 & 0,63 & 0,26 & 0,79 & 1,68 \\
\hline Copaifera langsdorffii Desf. (Caesalpiniaceae) & 3 & 0,24 & 0,61 & 0,79 & 1,64 \\
\hline Terminalia brasiliensis Raddi (Combretaceae) & 4 & 0,32 & 0,41 & 0,79 & 1,52 \\
\hline Diospyros hispida A. DC. (Ebenaceae) & 8 & 0,63 & 0,26 & 0,53 & 1,42 \\
\hline Erythroxylum dafnites Mart. (Erythroxylaceae) & 2 & 0,16 & 0,53 & 0,53 & 1,22 \\
\hline Machaerium aculeatum Raddi (Fabaceae) & 4 & 0,32 & 0,07 & 0,79 & 1,18 \\
\hline Heisteria ovata Benth. (Olacaceae) & 3 & 0,24 & 0,12 & 0,79 & 1,15 \\
\hline Myrcia rostrata DC. (Myrtaceae) & 3 & 0,24 & 0,08 & 0,53 & 0,85 \\
\hline Ormosia arborea (Vell.) Benth. (Fabaceae) & 1 & 0,08 & 0,48 & 0,26 & 0,82 \\
\hline Tabebuia sp. (Bignoniaceae) & 2 & 0,16 & 0,07 & 0,53 & 0,75 \\
\hline Ocotea minarum (Ness) Mez (Lauraceae) & 1 & 0,08 & 0,37 & 0,26 & 0,72 \\
\hline Myrsine umbellata (Mart.) Mez (Myrsinaceae) & 1 & 0,08 & 0,35 & 0,26 & 0,69 \\
\hline Jacaranda rufa Manso (Bignoniaceae) & 2 & 0,16 & 0,23 & 0,26 & 0,65 \\
\hline Bauhinia rufa (Bong.) Steud. (Caesalpiniaceae) & 1 & 0,08 & 0,29 & 0,26 & 0,64 \\
\hline Machaerium villosum Vog. (Fabaceae) & 1 & 0,08 & 0,22 & 0,26 & 0,56 \\
\hline Miconia affinis DC. (Melastomataceae) & 2 & 0,16 & 0,07 & 0,26 & 0,50 \\
\hline Machaerium nictitans (Vell.) Benth (Fabaceae) & 1 & 0,08 & 0,13 & 0,26 & 0,48 \\
\hline Brosimum gaudichaudii Tréc. (Moraceae) & 1 & 0,08 & 0,13 & 0,26 & 0,47 \\
\hline Agonandra brasiliensis Benth. \& Hook. F. (Opiliaceae) & 2 & 0,16 & 0,02 & 0,26 & 0,44 \\
\hline Cecropia pachystachya Tréc. (Cecropiaceae) & 1 & 0,08 & 0,08 & 0,26 & 0,42 \\
\hline Miconia albicans (Sw.) Triana (Melastomataceae) & 1 & 0,08 & 0,08 & 0,26 & 0,42 \\
\hline Cheiloclinium cognatum (Miers.) A.C. Smith (Hippocrateaceae) & 1 & 0,08 & 0,05 & 0,26 & 0,39 \\
\hline
\end{tabular}


Tabela 1 (continuação)

\begin{tabular}{|c|c|c|c|c|c|}
\hline Espécie/Família & $\mathrm{N}$ & D. R. & Do. R. & F. R. & IVI \\
\hline Zanthoxyllum rhoifolium Lamb. (Rutaceae) & 1 & 0,08 & 0,03 & 0,26 & 0,38 \\
\hline Luehea grandiflora Mart. \& Zucc. (Tiliaceae) & 1 & 0,08 & 0,03 & 0,26 & 0,38 \\
\hline Styrax camporum Pohl (Styracaceae) & 1 & 0,08 & 0,03 & 0,26 & 0,38 \\
\hline Ocotea puchella Mart. (Lauraceae) & 1 & 0,08 & 0,03 & 0,26 & 0,37 \\
\hline Rudgea viburnoides (Cham.) Benth. (Rubiaceae) & 1 & 0,08 & 0,02 & 0,26 & 0,37 \\
\hline Machaerium acutifolium Vogel (Fabaceae) & 1 & 0,08 & 0,02 & 0,26 & 0,36 \\
\hline Xylopia sericea A. St.-Hil. (Annonaceae) & 1 & 0,08 & 0,01 & 0,26 & 0,36 \\
\hline Aniba heringerii Vatt. (Lauraceae) & 1 & 0,08 & 0,01 & 0,26 & 0,36 \\
\hline
\end{tabular}

A diferença entre as populações estabelecidas nos dois estratos fornece indicações das variações temporais nas condições ambientais para as espécies em regeneração (Guariguata 2000; Mesquita 2000; Guariguata \& Ostertag 2001). Vários estudos enfatizam que o processo de regeneração é fortemente influenciado pelo histórico de uso, que afeta, de maneira diferenciada, os recursos disponíveis para os indivíduos que poderão se estabelecer em uma área (Aide et al. 1995; Mizrahi et al. 1997; Holl 1999; Pascarella et al. 2000).

A comparação entre os dois estratos permitiu observar que as espécies comuns não apresentaram a mesma ordenação em relação ao IVI (Tab. 1 e Tab. 2). Isso parece ocorrer especialmente em função da maior densidade dessas espécies comuns no estrato regenerativo.

A espécie com maior valor de IVI no estrato regenerante foi Piptocarpha macropoda $(12,92 \%$ do IVI total), que, apesar de não ter apresentado maiores valores de densidade e freqüência relativas, apresentou alta taxa de ocupação da área (dominância relativa). P. macropoda é considerada pioneira (Araújo et al. 1997; Felfili 1997; Felfili \& Abreu 1999; Nunes et al. 2003), ocupando, freqüentemente, o sub-bosque das matas, em áreas perturbadas. De acordo com Felfili \& Abreu (1999), P. macropoda apresenta maior densidade e indivíduos com maior estatura em condições de perturbação, como clareiras ou borda de matas, sugerindo sua presença como início de sucessão.

Ocotea corymbosa, a segunda em valor de importância no estrato de regeneração, destacou-se pela maior freqüência relativa, aparecendo em $83,3 \%$ das parcelas analisadas. No estrato arbóreo, esta espécie apresentou valores relativos de densidade, dominância e freqüência inferiores aos observados no estrato regenerativo, aparecendo em $23^{\circ}$ lugar no IVI. Segundo Nunes et al. (2003), O. corymbosa é considerada clímax exigente de luz (e de acordo com
Gandolfi et al. 1995, secundária inicial), o que reforça a condição inicial de sucessão do fragmento estudado, decorrente da permanente ação antrópica. Sua presença em $83,3 \%$ das parcelas revela que o ambiente tem favorecido o recrutamento de indivíduos dessa espécie. Por outro lado, sua baixa dominância no estrato arbóreo sugere que estes indivíduos permanecem no sub-bosque, até que sejam criadas condições de luminosidade que favoreçam competitivamente seu crescimento. Nunes et al. (2003) reforçam, ainda, que os indivíduos dessa espécie podem atingir grande porte, com possibilidade de ocupar o dossel da floresta.

Casearia grandiflora e Xylopia aromatica apresentaram altos valores de dominância relativa e, por isso, obtiveram maior IVI que Ocotea spixiana, que exibiu número de indivíduos, densidade e freqüência relativas maiores que as primeiras. Entretanto, estas três espécies demonstram grande potencial para ocupar o estrato superior da mata estudada, caso as condições ambientais se tornem favoráveis. C. grandiflora apareceu em terceiro lugar em IVI nos dois estratos. Entretanto, enquanto apresentou o segundo maior valor de dominância relativa no estrato regenerativo, esse valor está entre os mais baixos do estrato arbóreo. A explicação para a grande importância desta espécie no estrato arbóreo está nos valores relativos de densidade e freqüência, que são os maiores, se comparados às outras espécies deste estrato.

Uma importante observação deve ser feita com relação à presença de Dalbergia nigra e Senna macranthera, que são espécies citadas para formações da Mata Atlântica, e foram observadas na comunidade estudada. Estas espécies foram introduzidas no Parque para promover a arborização das áreas gramadas e ao longo das estradas. Entretanto, suas sementes estão sendo dispersas até a área da floresta nativa, onde encontram ambiente favorável para seu estabelecimento. De acordo com Cielo Filho 
Tabela 2. Estrutura fitossociológica do estrato arbóreo da floresta estacional semidecídua do Parque do Sabiá, Uberlândia, MG, em ordem decrescente do Índice de Valor de Importância. (N= número de indivíduos amostrados; D.R.= densidade relativa; Do.R.= dominância relativa; F.R.= freqüência relativa; IVI= Índice de Valor de Importância).

\begin{tabular}{|c|c|c|c|c|c|}
\hline Espécie/Família & $\mathrm{N}$ & D. R. & Do. R. & F. R. & IVI \\
\hline Tapirira obtusa (Benth.) J.D. Mitch. (Anacardiaceae) & 48 & 7,55 & 10,92 & 5,06 & 23,53 \\
\hline Virola sebifera Aubl. (Myristicaceae) & 61 & 9,59 & 5,09 & 7,17 & 21,85 \\
\hline Casearia grandiflora Camb. (Flacourtiaceae) & 63 & 9,91 & 2,91 & 7,81 & 20,62 \\
\hline Inga vera Willd. (Mimosaceae) & 51 & 8,02 & 2,72 & 7,38 & 18,13 \\
\hline Cryptocaria aschersoniana Mez (Lauraceae) & 27 & 4,25 & 8,57 & 4,01 & 16,83 \\
\hline Piptocarpha macropoda (DC.) Baker. (Asteraceae) & 38 & 5,97 & 4,52 & 6,12 & 16,61 \\
\hline Copaifera langsdorffii Desf. (Caesalpiniaceae) & 11 & 1,73 & 12,00 & 2,11 & 15,84 \\
\hline Astronium nelson-rosae D.A. Santin (Anacardiaceae) & 23 & 3,62 & 6,58 & 3,80 & 14,00 \\
\hline Micropholis venulosa (Mart. \& Eichl.) Pierre (Sapotaceae) & 23 & 3,62 & 4,70 & 4,01 & 12,33 \\
\hline Amaioua guianensis Aubl. (Rubiaceae) & 29 & 4,56 & 1,50 & 4,22 & 10,28 \\
\hline Heisteria ovata Benth. (Olacaceae) & 23 & 3,62 & 1,23 & 4,22 & 9,06 \\
\hline Callisthene major Mart. (Vochysiaceae) & 18 & 2,83 & 3,52 & 2,53 & 8,88 \\
\hline Annona cacans Warm. (Annonaceae) & 8 & 1,26 & 4,88 & 1,69 & 7,83 \\
\hline Terminalia brasiliensis Raddi (Combretaceae) & 17 & 2,67 & 2,12 & 2,74 & 7,54 \\
\hline Didymopanax morototoni (Aubl.) Decne. \& Planch. (Araliaceae) & 10 & 1,57 & 2,37 & 2,11 & 6,05 \\
\hline Ocotea spixiana (Ness) Mez (Lauraceae) & 7 & 1,10 & 3,40 & 1,48 & 5,98 \\
\hline Miconia sellowiana Naud. (Melastomataceae) & 9 & 1,42 & 1,38 & 1,90 & 4,70 \\
\hline Aspidosperma discolor A. DC. (Apocynaceae) & 10 & 1,57 & 1,62 & 1,48 & 4,67 \\
\hline Cordia sellowiana Cham. (Boraginaceae) & 10 & 1,57 & 0,72 & 2,11 & 4,40 \\
\hline Cheiloclinium cognatum (Miers.) A.C. Smith (Hippocrateaceae) & 13 & 2,04 & 0,28 & 1,90 & 4,22 \\
\hline Ixora gardneriana Benth. (Rubiaceae) & 8 & 1,26 & 1,07 & 1,69 & 4,01 \\
\hline Qualea jundiahy Warm. (Vochysiaceae) & 6 & 0,94 & 1,95 & 1,05 & 3,95 \\
\hline Ocotea corymbosa (Miers.) Mez (Lauraceae) & 7 & 1,10 & 1,52 & 1,05 & 3,68 \\
\hline Ormosia arborea (Vell.) Benth (Fabaceae) & 8 & 1,26 & 0,90 & 1,48 & 3,63 \\
\hline Maprounea guianensis (Aubl.) M. Arg. (Euphorbiaceae) & 10 & 1,57 & 0,58 & 1,48 & 3,63 \\
\hline Miconia cuspidata Naud. (Melastomataceae) & 4 & 0,63 & 1,77 & 0,84 & 3,25 \\
\hline Coussarea hydrangeaefolia Benth. \& Hook. f. (Rubiaceae) & 8 & 1,26 & 0,44 & 1,48 & 3,18 \\
\hline Duguetia lanceolata A. St.-Hil. (Annonaceae) & 6 & 0,94 & 0,74 & 1,05 & 2,74 \\
\hline Lamanonea ternata Vell. (Cunnoniaceae) & 4 & 0,63 & 0,93 & 0,84 & 2,40 \\
\hline Hirtella glandulosa Spreng. (Chrysobalanaceae) & 3 & 0,47 & 0,77 & 0,63 & 1,87 \\
\hline Xylopia aromatica (Lamb.) Mart. (Annonaceae) & 4 & 0,63 & 0,30 & 0,84 & 1,77 \\
\hline Machaerium oblongifolium Vog. (Fabaceae) & 3 & 0,47 & 0,55 & 0,63 & 1,65 \\
\hline Siparuna guianensis Aubl. (Monimiaceae) & 4 & 0,63 & 0,14 & 0,84 & 1,61 \\
\hline Cassia ferruginea (Schrad.) Schrad. ex DC. (Caesalpiniaceae) & 3 & 0,47 & 0,48 & 0,63 & 1,58 \\
\hline Stryrax camporum Pohl (Styracaceae) & 2 & 0,31 & 0,77 & 0,42 & 1,51 \\
\hline Vochysia tucanorum Mart. Vochysiaceae & 2 & 0,31 & 0,72 & 0,42 & 1,45 \\
\hline Hymenaea courbaril L. (Caesalpiniaceae) & 1 & 0,16 & 1,07 & 0,21 & 1,44 \\
\hline Pseudolmedia laevigata Tréc. (Moraceae) & 4 & 0,63 & 0,12 & 0,63 & 1,38 \\
\hline Pera glabrata (Schott.) M. Arg. (Euphorbiaceae) & 2 & 0,31 & 0,45 & 0,42 & 1,19 \\
\hline Apuleia mollaris (Mart.) Spreng. (Fabaceae) & 1 & 0,16 & 0,79 & 0,21 & 1,16 \\
\hline Bauhinia rufa (Bong.) Steud. (Caesalpiniaceae) & 3 & 0,47 & 0,04 & 0,63 & 1,14 \\
\hline Myrcia rostrata DC. (Myrtaceae) & 4 & 0,63 & 0,08 & 0,42 & 1,13 \\
\hline Matayba guianensis Aubl. (Sapindaceae) & 3 & 0,47 & 0,21 & 0,42 & 1,10 \\
\hline Protium heptaphyllum (Aubl.) March. (Burseraceae) & 2 & 0,31 & 0,30 & 0,42 & 1,04 \\
\hline Não identificada 2 & 2 & 0,31 & 0,22 & 0,42 & 0,95 \\
\hline Hirtella gracilipes (Hook. f.) (Chrysobalanaceae) & 2 & 0,31 & 0,21 & 0,42 & 0,94 \\
\hline Qualea grandiflora Mart. (Vochysiaceae) & 2 & 0,31 & 0,16 & 0,42 & 0,89 \\
\hline Zanthoxylum rhoifolium Lamb. (Rutaceae) & 2 & 0,31 & 0,10 & 0,42 & 0,83 \\
\hline Garcinia gardneriana (Planch. \& Triana) Zappi (Clusiaceae) & 2 & 0,31 & 0,08 & 0,42 & 0,82 \\
\hline Tabebuia impetiginosa (Mart. ex DC.) Standl. (Bignoniaceae) & 2 & 0,31 & 0,05 & 0,42 & 0,79 \\
\hline Inga laurina (Sw.) Willd. (Mimosaceae) & 2 & 0,31 & 0,05 & 0,42 & 0,78 \\
\hline Myrcia tomentosa (Aubl.) DC. (Myrtaceae) & 2 & 0,31 & 0,03 & 0,42 & 0,76 \\
\hline Pterodon pubecens (Benth.) Benth. (Fabaceae) & 1 & 0,16 & 0,36 & 0,21 & 0,72 \\
\hline Não identificada 7 & 1 & 0,16 & 0,35 & 0,21 & 0,72 \\
\hline
\end{tabular}


Tabela 2 (continuação)

\begin{tabular}{|c|c|c|c|c|c|}
\hline Espécie/Família & $\mathrm{N}$ & D. R. & Do. R. & F. R. & IVI \\
\hline Não identificada 3 & 1 & 0,16 & 0,20 & 0,21 & 0,56 \\
\hline Senna macranthera (Collad.) I. \& B. (Caesalpiniaceae) & 1 & 0,16 & 0,10 & 0,21 & 0,47 \\
\hline Lauraceae 1 & 1 & 0,16 & 0,06 & 0,21 & 0,42 \\
\hline Myrtaceae 1 & 1 & 0,16 & 0,04 & 0,21 & 0,41 \\
\hline Não identificada 6 & 1 & 0,16 & 0,04 & 0,21 & 0,41 \\
\hline Não identificada 5 & 1 & 0,16 & 0,04 & 0,21 & 0,40 \\
\hline Myrsine guianensis (Aubl.) Kuntz. (Myrsinaceae) & 1 & 0,16 & 0,04 & 0,21 & 0,40 \\
\hline Rudgea viburnoides (Cham.) Benth. (Rubiaceae) & 1 & 0,16 & 0,03 & 0,21 & 0,40 \\
\hline Não identificada 4 & 1 & 0,16 & 0,03 & 0,21 & 0,40 \\
\hline Cardiopetalum calophyllum Schlecht. (Annonaceae) & 1 & 0,16 & 0,02 & 0,21 & 0,39 \\
\hline Luehea grandiflora Mart. \& Zucc. (Tiliaceae) & 1 & 0,16 & 0,02 & 0,21 & 0,38 \\
\hline Não identificada 1 & 1 & 0,16 & 0,01 & 0,21 & 0,38 \\
\hline Faramea hyacinthina Mart. (Rubiaceae) & 1 & 0,16 & 0,01 & 0,21 & 0,38 \\
\hline Plathymenia reticulata Benth. (Mimosaceae) & 1 & 0,16 & 0,01 & 0,21 & 0,38 \\
\hline Cupania vernalis Camb. (Sapindaceae) & 1 & 0,16 & 0,01 & 0,21 & 0,38 \\
\hline Erythroxylum daphnites Mart. (Erythroxylaceae) & 1 & 0,16 & 0,01 & 0,21 & 0,38 \\
\hline Platypodium elegans Vogel (Fabaceae) & 1 & 0,16 & 0,01 & 0,21 & 0,38 \\
\hline
\end{tabular}

\& Santin (2002), a introdução de espécies exóticas e nativas de outras regiões nas proximidades de pequenos fragmentos isolados resulta no aumento da competição com as espécies nativas. Na maioria das vezes, o estabelecimento de exóticas é favorecido devido às mudanças nas condições ambientais decorrentes da descaracterização estrutural dos remanescentes florestais.

Em resumo, espécies com grande importância no estrato regenerativo, como Ocotea corymbosa, Xylopia aromatica, Ocotea spixiana, Siparuna guianensis, Annona cacans e Maprounea guianensis, apresentaram valores relativos de densidade, dominância e freqüência inferiores no estrato arbóreo adulto. Por outro lado, Tapirira obtusa, Cryptocaria aschersoniana, Copaifera langsdorffii, Astronium nelson-rosae, Micropholis venulosa e Amaioua guianensis tiveram valores relativos mais expressivos, e, conseqüentemente, maior IVI no levantamento do estrato arbóreo. $\mathrm{O}$ alto valor de importância de espécies secundárias no estrato arbóreo, em detrimento das espécies iniciais, é freqüente em florestas estacionais semidecíduas, o que está diretamente relacionado a seu histórico de perturbação (Gandolfi et al. 1995; Ivanauskas et al. 1999). A presença de espécies tardias de grande porte no estrato arbóreo, sem a correspondência de abundância no estrato regenerante, sugere que as perturbações atingem diretamente o estrato inferior.

Para que uma população de espécie arbórea tenha sucesso no seu processo de manutenção numa determinada comunidade florestal, espera-se encontrar grande quantidade de indivíduos regenerantes. Isso pode ser considerado uma estratégia reprodutiva, já que muitos não atingirão a fase adulta, devido à ação de inúmeros processos interativos, como predação e competição. Esses processos são mais intensos nos ambientes antropizados.

O dossel da floresta estacional do Parque do Sabiá é composto de árvores que, provavelmente, possuem dezenas de anos e a formação de clareiras, naturais ou antrópicas, tem estimulado o estabelecimento de espécies pioneiras e o crescimento de grupos de regenerantes. De acordo com Silvertown \& Doust (1993), em comunidades nas quais as populações apresentam indivíduos de grande longevidade, o recrutamento é um evento freqüente. Entretanto, os indivíduos regenerantes tendem a ter maior taxa de mortalidade que os adultos, de forma que as taxas de recrutamento e mortalidade se apresentam positivamente correlacionadas nas populações, assim como na comunidade como um todo.

Quanto às espécies mais importantes do estrato arbóreo, Tapirira obtusa, Cryptocaria aschersoniana e Copaifera langsdorffii apresentaram proporção entre regenerantes e adultos menor que 1:1 (Tab. 3), sugerindo que as espécies mais importantes do dossel atual não têm tido sucesso regenerativo dentro da comunidade. Assim, a morte dos adultos poderá significar a extinção local destas espécies (consideradas tardias), devido à ausência de recrutamento, e resultar na mudança florística da formação. 
Tabela 3. Proporção entre indivíduos regenerantes e adultos na floresta estacional semidecídua do Parque do Sabiá, Uberlândia, MG.

\begin{tabular}{|c|c|c|c|}
\hline Espécies & $\begin{array}{l}\text { Densidade absoluta de indivíduos } \\
\left.\text { regenerantes (ind. } \mathrm{ha}^{-1}\right)(\mathrm{A})\end{array}$ & $\begin{array}{c}\text { Densidade absoluta de indivíduos } \\
\text { adultos (ind. } \text { ha }^{-1} \text { ) (B) }\end{array}$ & Proporção (A.B $\left.{ }^{-1}\right)$ \\
\hline 1. Piptocarpha macropoda* & 623,3 & 54,3 & 11,5 \\
\hline 2. Ocotea corymbosa & 630,0 & 10,0 & 63,0 \\
\hline 3. Casearia grandiflora* & 326,7 & 90,0 & 3,6 \\
\hline 4. Xylopia aromatica & 216,7 & 5,7 & 38,0 \\
\hline 5. Ocotea spixiana & 343,3 & 10,0 & 34,3 \\
\hline 6. Siparuna guianensis & 206,7 & 5,7 & 36,3 \\
\hline 7. Annona cacans & 236,7 & 11,4 & 20,8 \\
\hline 8. Maprounea guianensis & 190,0 & 14,3 & 13,3 \\
\hline 9. Inga vera* & 113,3 & 72,8 & 1,5 \\
\hline 10. Virola sebifera* & 113,3 & 87,1 & 1,3 \\
\hline 11. Tapirira obtusa & 23,3 & 68,6 & 0,3 \\
\hline 12. Cryptocaria aschersoniana & 20,0 & 38,6 & 0,5 \\
\hline 13. Copaifera langsdorffii & 10,0 & 15,7 & 0,6 \\
\hline 14. Astronium nelson-rosae & 50,0 & 32,9 & 1,5 \\
\hline 15. Micropholis venulosa & 130,0 & 32,9 & 3,9 \\
\hline 16. Amaioua guianensis & 100,0 & 41,4 & 2,4 \\
\hline
\end{tabular}

1-10: dez espécies mais importantes do estrato regenerativo; 11-16: espécies mais importantes somente no estrato arbóreo; *espécies comuns entre as dez mais importantes dos estratos arbóreo e regenerativo.

De acordo com Swaine \& Hall (1988), inúmeras comunidades tropicais apresentam populações abundantes no dossel atual, porém, são raras ou mesmo ausentes na fase plântulas e plantas jovens. As espécies do estrato arbóreo que naturalmente ocorrem em baixa densidade e possuem poucos indivíduos recrutados no estrato inferior, estão mais susceptíveis à extinção local, devido às variações ambientais e demográficas, problemas genéticos e catástrofes naturais (Nascimento et al. 1999).

O índice de similaridade florística entre os dois estratos estudados foi de $61 \%$. Embora seja um alto valor, ele está baseado em registros de presença/ ausência de espécies, sem levar em conta o número de indivíduos, o que nos parece mais relevante quando se trata do potencial de regeneração natural de uma comunidade. Por essa razão, a comparação dos resultados quantitativos, para cada espécie, em cada estrato, assume maior relevância. A alta proporção de indivíduos em regeneração das espécies mais importantes do estrato regenerativo sugere que tais espécies poderão representar a futura comunidade arbórea (Tab. 3). De forma geral, o dossel de uma floresta muda à medida que as árvores crescem e morrem e outras as substituem (Fonseca e Rodrigues 2000). De acordo com esses autores, tal estrutura se forma a partir do equilíbrio dinâmico, representado pela presença de três fases: fase de clareira, fase de construção (floresta jovem) e fase madura. Assim, a comunidade deverá sofrer mudanças na sua composição florística ao longo do processo de sucessão, devido ao ingresso das espécies pertencentes aos primeiros estágios sucessionais (encontradas no estrato regenerativo), com conseqüente substituição das espécies de estágios mais avançados (encontradas no estrato arbóreo).

É importante ressaltar que o método adotado para essa comparação considera que os indivíduos encontram-se distribuídos de forma homogênea no ambiente, o que não é verdadeiro nas formações vegetais tropicais. Sabe-se que a disposição das plantas no ambiente está associada a uma variedade de fatores, os quais influenciam na forma de colonização de um ambiente (Shmida \& Ellner 1984). Assim, existe a possibilidade de que espécies que se estabelecem de forma agrupada, influenciadas por fatores como a proximidade à planta-mãe, não tenham sido amostradas satisfatoriamente, devido ao método de amostragem (30 parcelas para o estrato regenerante e 70 parcelas para o estrato arbóreo), como também sugerido por Barreira et al. (2002).

Este trabalho traz evidências de que os níveis de perturbação antrópica resultante das medidas inadequadas de manejo aplicadas no passado têm afetado diretamente o processo de regeneração natural na área de estudo. Esta influência se apresenta de forma mais clara sobre a densidade e a distribuição das espécies na área como um todo. Mesmo 
apresentando um alto índice de similaridade florística entre os dois estratos estudados $(61 \%)$, existem diferenças marcantes entre eles quanto à abundância das espécies mais importantes. Sugere-se um monitoramento na área, por meio de estudos de dinâmica de comunidade nos dois estratos, como forma de diagnosticar e propor ações de manejo que minimizem os efeitos da perturbação passada sobre a possível extinção local de espécies nativas importantes na área de estudo.

\section{Agradecimentos}

Os autores agradecem ao Prof. Dr. Paulo Eugênio de Oliveira, pela revisão do Abstract; à Dra. Giselda Durigan, pelas valiosas sugestões; aos revisores anônimos, pelas valiosas críticas e sugestões.

\section{Referências bibliográficas}

Aide, T.M.; Zimmerman, J.K.; Herrera, L.; Rosario, M. \& Serrano, M. 1995. Forest recovery in abandoned pastures in Puerto Rico. Forest Ecology and Management 77: 77-86.

Aide, T.M.; Zimmerman, J.K.; Pascarella, J.B.; Rivera, L. \& Marcano-Veja, H. 2000. Forest regeneration in a chronosequence of tropical abandoned pastures: implications for restoration ecology. Restoration Ecology 8(4): 328-338.

Aizen, M.A. \& Feinsinger, P. 1994. Forest fragmentation, pollination, and plant reproduction in Chaco dry forest, Argentina. Ecology 75: 330-351.

Araújo, G.M.; Guimarães, A.J.M. \& Nakajima, J.N. 1997. Fitossociologia de um remanescente de mata mesófila semidecídua urbana, Bosque John Kennedy, Araguari, MG, Brasil. Revista Brasileira de Botânica 20: 67-77.

Baccaro, C.A.D. 1989. Estudos geomorfológicos do Município de Uberlândia. Sociedade e Natureza 1(1): 17-21.

Barreira, S.; Scolforo, J.R.S.; Botelho, S.A. \& Mello, J.M. 2002. Estudo da estrutura da regeneração natural e da vegetação adulta de um cerrado senso stricto para fins de manejo florestal. Scientia Forestalis 61: 64-78.

Brower, J.E.; Zar, J.H. \& von Ende, C.N. 1997. Field and Laboratory Methods for General Ecology. $4^{\text {th }}$. ed., Boston, WCB MacGraw-Hill.

Brown, S. \& Lugo, A.E. 1990. Tropical secondary forests. Journal of Tropical Ecology 6(1): 1-32.

Cielo Filho, R. \& Santin, D.A. 2002. Estudo florístico e fitossociológico de um fragmento florestal urbano Bosque dos Alemães, Campinas, SP. Revista Brasileira de Botânica 25(3): 291-301.

Cronquist, A. 1988. The evolution and classification of flowering plants. $2^{\text {nd }}$. ed. Bronx, New York Botanical Garden.
Dislich, R.; Cersósimo, L. \& Mantovani, W. 2001. Análise da estrutura de fragmentos florestais no Planalto Paulistano - SP. Revista Brasileira de Botânica 24(3): 321-332.

Durigan, G.; Franco, G.A.D.C.; Saito, M. \& Baitello, J.B. 2000. Estrutura e diversidade do componente arbóreo da floresta na Estação Ecológica dos Caetetus, Gália, SP. Revista Brasileira de Botânica 23(4): 371-383.

Durigan, G.; Nishikawa, D.L.L.; Rocha, E.; Silveira, E.R.; Pulitano, F.M.; Regalado, L.B.; Carvalhaes, M.A.; Paranaguá, P.A. \& Ranieri, V.E.L. 2002. Caracterização de dois estratos da vegetação em uma área de cerrado no município de Brotas, SP, Brasil. Acta Botanica Brasilica 16(3): 251-262.

Felfili, J.M. 1997. Dynamics of the natural regeneration in the Gama gallery forest in Central Brazil. Forest Ecology and Management 91: 235-245.

Felfili, J.M. \& Abreu. H.M. 1999. Regeneração natural de Roupala montana Aubl., Piptocarpha macropoda Back. e Persea fusca Mez. em quatro condições ambientais na mata de galeria do Gama - DF. CERNE 5(2): 125-132.

Felfili, J.M.; Ribeiro, J.F.; Fagg, C.W. \& Machado, J.W.B. 2000. Recuperação de matas de galeria. Embrapa Cerrado Série Técnica 21(1): 45-45.

Fonseca, R.C.B. \& Rodrigues, R.R. 2000. Análise estrutural e aspectos do mosaico sucessional de uma floresta semidecídua em Botucatu, SP. Scientia Forestalis 57: 27-43.

Galetti, M.; Alves-Costa, C.P. \& Cazetta, E. 2003. Effects of forest fragmentation, anthropogenic edges and fruit colour on the consumption of ornithocoric fruits. Biological Conservation 111: 269-273.

Gandolfi, S.; Leitão Filho, H.F. \& Bezerra, C.L.F. 1995. Levantamento florístico e caráter sucessional das espécies arbustivo-arbóreas de uma floresta mesófila semidecídua no município de Guarulhos, SP. Revista Brasileira de Biologia 55(4): 753-767.

Grapow, L.C. \& Blasi, C.A. 1998. Comparison of the urban flora of different phytoclimatic regions in Italy. Global Ecology and Biogeography Letters 7: 367-378.

Guariguata, M.R. 2000. Seed and seedling ecology of the tree species in neotropical secondary forests: management implications. Ecological Applications 10(1): 145-154.

Guariguata, M.R. \& Ostertag, R. 2001. Neotropical secondary forest succession: changes in structural and functional characteristics. Forest Ecology and Management 148: 185-206.

Guilherme, F.A.G.; Nakajima, J.N.; Lima, C.A.P. \& Vanini, A. 1998. Fitofisionomias e a flora lenhosa nativa do Parque do Sabiá, Uberlândia, MG. Daphne 8(2): 17-30.

Holl, K.D. 1999. Factors limiting tropical rain forest regeneration in abandoned pasture: seed rain, seed germination, microclimate and soil. Biotropica 31(2): 229-252.

Ivanauskas, N.M.; Rodrigues, R.R. \& Nave, A.G. 1999. Fitossociologia de um trecho de floresta estacional semidecidual em Itatinga, São Paulo, Brasil. Scientia Florestalis 56: 83-99. 
Mesquita, R.D.G. 2000. Management of advanced regeneration in secondary forests of the Brazilian Amazon. Forest Ecology and Management 130(1-3): 131-140.

Mizrahi, A.; Prado, J.M.R. \& Jimenez-Osornio, J. 1997. Composition, structure and management potential of secondary dry tropical vegetation in two abandoned plantations of Yucatan, Mexico. Forest Ecology and Management 96(3): 273-282.

Nascimento, H.E.M.; Dias, A.S.; Tabanez, A.A.J. \& Viana, V.M. 1999. Estrutura e dinâmica de populações arbóreas de um fragmento de Floresta Estacional Semidecidual na região de Piracicaba, São Paulo. Revista Brasileira de Botânica 59(2): 329-342.

Nunes, Y.R.F.; Mendonça, A.V.R.; Botezelli, L.; Machado, E.L.M. \& Oliveira Filho, A.T. 2003. Variações da fisionomia, diversidade e composição de guildas da comunidade arbórea em um fragmento de floresta semidecidual em Lavras, MG. Acta Botanica Brasilica 17(2): 213-229.

Pagano, S.N. \& Leitão Filho, H.F. 1987. Composição florística do estrato arbóreo de mata mesófila semidecídua no município de Rio Claro, Estado de São Paulo. Revista Brasileira de Botânica 10: 37-47.

Pascarella, J.B.; Aide, T.M. \& Serrano, M.I. 2000. Land-use history and forest regeneration in the Cayey Mountains, Puerto Rico. Ecosystems 3(3): 217-228.

Paula, S.A. \& Lemos Filho, J.P. 2001. Dinâmica do dossel em mata semidecídua no perímetro urbano de Belo Horizonte, MG. Revista Brasileira de Botânica 24(4 supl.): 545-551.

Pereira, I.M.; Andrade, L.A.; Costa, J.R.M. \& Dias, J.M. 2001. Regeneração natural em um remanescente de Caatinga sob diferentes níveis de perturbação, no Agreste Paraibano. Acta Botânica Brasílica 15(3): 413-426.

Ribeiro, J.F. \& Walter, B.M.T. 1998. Fitofisionomias do bioma Cerrado. Pp. 89-166. In: S.M. Sano \& S.P. Almeida (eds.). Cerrado: ambiente e flora. Planaltina, EMBRAPA CPAC.
Rosa, A.G. \& Schiavini 2006. Estrutura da comunidade arbórea em um remanescente florestal urbano (Parque do Sabiá, Uberlândia, MG). Bioscience Journal 22(1): 151-162.

Rosa, R.; Lima, S.C. \& Assunção, W.L. 1991. Abordagem preliminar das condições climáticas de Uberlândia (MG). Sociedade e Natureza 3(5-6): 91-108.

Santos, T. \& Tellería, J.L. 1994. Influence of forest fragmentation on seed consumption and dispersal of spanish juniper Juniperus thurifera. Biological Conservation 70: 129-134.

Saunders, D.A.; Hobbs, R.J. \& Margules, C.R. 1991. Biological consequences of ecosystem fragmentation: a review. Conservation Biology 5: 18-532.

Shepherd, G.J. 1995. FITOPAC 1: Manual do Usuário. Campinas, Universidade Estadual de Campinas.

Shmida, A. \& Ellner, S. 1984. Coexistence of plant species with similar niches. Vegetatio 58: 29-55.

Silverstown, J.W. \& Doust, J.L. 1993. Introduction to plant population biology. $3^{\text {rd }}$ ed. Blackwell Science.

Swaine, M.D. \& Hall, J.B. 1988. The mosaic theory of forest regeneration and the determination of forest composition in Ghana. Journal of Tropical Ecology 4: 253-269.

Tabarelli, M. \& Mantovani, W. 1999. A regeneração de um floresta tropical montana após corte e queima (São Paulo - Brasil). Revista Brasileira de Biologia 59(2): 239-250.

Viana, V.M.; Tabanez, A.A.J. \& Martinez, J.L.A. 1992. Restauração e manejo de fragmentos florestais. Pp. 400-407. In: II Congresso Nacional sobre Essências Nativas. São Paulo, Instituto Florestal de São Paulo.

Werneck, M.S.; Pedralli, G.; Koenig, R. \& Giseke, L.F. 2000. Florística e estrutura de três trechos de uma floresta semidecidual na Estação Ecológica do Tripuí, Ouro Preto, MG. Revista Brasileira de Botânica 23(1): 97-106. 